TRANSACTIONS OF THE

AMERICAN MATHEMATICAL SOCIETY

Volume 362, Number 2, February 2010, Pages 661-672

S 0002-9947(09)04673-X

Article electronically published on September 18, 2009

\title{
NON-UNIQUE SOLUTIONS TO BOUNDARY VALUE PROBLEMS FOR NON-SYMMETRIC DIVERGENCE FORM EQUATIONS
}

\author{
ANDREAS AXELSSON
}

\begin{abstract}
We calculate explicitly solutions to the Dirichlet and Neumann boundary value problems in the upper half plane, for a family of divergence form equations having non-symmetric coefficients with a jump discontinuity. It is shown that the boundary equation method and the Lax-Milgram method for constructing solutions may give two different solutions when the coefficients are sufficiently non-symmetric.
\end{abstract}

\section{INTRODUCTION}

Recently, new techniques in harmonic analysis have been used to study boundary value problems (BVP's) for divergence form elliptic equations with non-symmetric or more general complex coefficients. In the half plane, for real but non-symmetric coefficients, $L_{p}$ solvability of the Dirichlet problem for sufficiently large $p$ was obtained by Kenig, Koch, Pipher and Toro [3], and $L_{p}$ solvability of the Neumann and regularity problems, for sufficiently small $p$, was proved by Kenig and Rule 44. In $\mathbf{R}^{n}$, two boundary equation methods have been studied by Alfonseca, Auscher, Axelsson, Hofmann and Kim [1] and by Auscher, Axelsson and Hofmann 2] where, among other things, these BVP's were proved to be well posed in $L_{2}$ for small complex $L_{\infty}$ perturbations of real symmetric coefficients.

Unlike the case of real symmetric coefficients, for general non-symmetric coefficients well-posedness of these classical BVP's may fail. In [3] and [4, the family

$$
A_{k}(x):=\left[\begin{array}{cc}
1 & k \operatorname{sgn}(x) \\
-k \operatorname{sgn}(x) & 1
\end{array}\right]
$$

of non-symmetric coefficient matrices with a jump at $x=0$ was studied and shown to provide counterexamples to well-posedness for certain values of the parameter $k \in \mathbf{R}$. More precisely, the following theorem was proved in [3. Theorem (3.2.1)] and [4, Appendix].

Theorem 1.1. Let $1<p<\infty$. The Dirichlet problem (Dir- $A_{k}, p$ ) fails to be well posed in the $\dot{H}^{1}$ sense if $k>\tan \left(\frac{\pi}{2 q}\right)$, where $1 / q=1-1 / p$. The regularity problem $\left(\right.$ Reg- $\left.A_{k}, p\right)$ fails to be well posed in the $\dot{H}^{1}$ sense if $k<-\tan \left(\frac{\pi}{2 p}\right)$. The Neumann problem $\left(\mathrm{Neu}-A_{k}, p\right)$ fails to be well posed in the $\dot{H}^{1}$ sense if $k>\tan \left(\frac{\pi}{2 p}\right)$.

In this paper, we demonstrate that one must be careful in specifying in what sense well-posedness is meant, when considering BVP's for non-symmetric coefficients. (The notion of well-posedness in the $\dot{H}^{1}$ sense is defined below.) Indeed,

Received by the editors September 14, 2007.

2000 Mathematics Subject Classification. Primary 35J25, 42A50.

(C)2009 American Mathematical Society 661

Reverts to public domain 28 years from publication 
Theorem 1.2 below shows that these BVP's can be well posed in an $L_{\infty}\left(L_{p}\right)$ sense, as defined below, without being well posed in the $\dot{H}^{1}$ sense.

To explain these results, we first need to introduce the notion of $\dot{H}^{1}$ solutions and $L_{\infty}\left(L_{p}\right)$ solutions to BVP's. We consider a given divergence form equation

$$
\operatorname{div} A(x) \nabla U(t, x)=0
$$

in the upper half plane $\mathbf{R}_{+}^{2}:=\left\{(t, x) \in \mathbf{R}^{2} ; t>0\right\}$, where $A=\left(a_{i j}\right)_{i, j=0,1} \in$ $L_{\infty}\left(\mathbf{R} ; \mathcal{L}\left(\mathbf{C}^{2}\right)\right)$ is a $t$-independent, complex and accretive coefficient matrix such that $\operatorname{Re}(A(x) v, v) \geq \kappa|v|^{2}$ for all $x \in \mathbf{R}, v \in \mathbf{C}^{2}$ and for some $\kappa>0$, and where $U$ satisfies one of the following prescribed boundary conditions.

- The Dirichlet problem (Dir- $A, p): U(0, \cdot)=u$, for a given function $u \in$ $L_{p}(\mathbf{R} ; \mathbf{C})$.

- The (Dirichlet) regularity problem $(\operatorname{Reg}-A, p): \partial_{1} U(0, \cdot)=u^{\prime}$, for a given function $u \in \dot{W}_{p}^{1}(\mathbf{R} ; \mathbf{C})$.

- The Neumann problem (Neu- $A, p): a_{00} \partial_{0} U(0, \cdot)+a_{01} \partial_{1} U(0, \cdot)=\phi$, for a given function $\phi \in L_{p}(\mathbf{R} ; \mathbf{C})$. This means that the conormal derivative of $U$ is prescribed.

Throughout this paper, $p$ denotes a fixed exponent such that $1<p<\infty$, and $q$ is the dual exponent. The regularity and Neumann problems can be thought of as BVP's for the gradient vector field $F(t, x)=F_{0} e_{0}+F_{1} e_{1}:=\nabla U(t, x)$, rather than for $U$ itself. Here $e_{0}$ denotes the vertical basis vector along the $t=x_{0}$-axis, and $e_{1}$ is the horizontal basis vector along the $x=x_{1}$-axis.

There are two classical methods for constructing a solution $U$ : the Lax-Milgram lemma and boundary equation methods.

$\dot{H}^{1}$ solutions. To solve a Dirichlet problem for a sufficiently smooth and localised boundary function $u(x)$, one first constructs a function $U_{1}$ in $\mathbf{R}^{2}$ such that $U_{1}(0, x)=u(x)$. Next, one uses the Lax-Milgram lemma to find a function $U_{2} \in \dot{H}_{0}^{1}\left(\mathbf{R}_{+}^{2}\right)$, which decays at infinity, such that $B\left(U_{2}, \psi\right)=\ell(\psi)$ for all $\psi \in \dot{H}_{0}^{1}\left(\mathbf{R}_{+}^{2}\right)$, where

$$
B\left(U_{2}, \psi\right):=\iint_{\mathbf{R}_{+}^{2}}\left(A(x) \nabla U_{2}(t, x), \nabla \psi(t, x)\right) d t d x
$$

and the given functional is $\ell(\psi):=\iint_{\mathbf{R}_{+}^{2}}\left(A(x) \nabla U_{1}(t, x), \nabla \psi(t, x)\right) d t d x$. Details of this construction for the unbounded domain $\mathbf{R}_{+}^{2}$ can be found in [4, Lemma 1.1]. The function $U:=U_{1}-U_{2}$ now solves equation (11) and has boundary trace $u$, in a weak sense.

We say that the BVP (Dir- $A, p)$ is well posed in the $\dot{H}^{1}$ sense if for all sufficiently smooth and localised $u$, the solution $U$ constructed above has quantitative bound

$$
\left\|N_{*}(U)\right\|_{L_{p}(\mathbf{R})} \leq C_{p}\|u\|_{L_{p}(\mathbf{R})} .
$$

Similarly, $(\operatorname{Reg}-A, p)$ is well posed in the $\dot{H}^{1}$ sense if for all sufficiently smooth and localised $u$, the solution $U$ constructed above has quantitative bound

$$
\left\|\widetilde{N}_{*}(\nabla U)\right\|_{L_{p}(\mathbf{R})} \leq C_{p}\left\|u^{\prime}\right\|_{L_{p}(\mathbf{R})} .
$$


Here

$$
\begin{aligned}
N_{*}(U)\left(x_{0}\right) & :=\sup _{\left|x-x_{0}\right|<t}|U(t, x)| \\
\text { and } \widetilde{N}_{*}(F)\left(x_{0}\right) & :=\sup _{\left|x-x_{0}\right|<t} t^{-1}\|F\|_{L_{2}(Q(t, x))},
\end{aligned}
$$

where $Q(t, x)$ denotes the square centered at $(t, x)$ with sidelength $t$, are the standard (modified) non-tangential maximal functions, and $C_{p}$ denotes a constant independent of $u$.

Turning to the Neumann problem, this is solved for a sufficiently smooth and localised boundary function $\phi(x)$ with $\int \phi=0$ by applying the Lax-Milgram lemma to obtain $U \in \dot{H}^{1}\left(\mathbf{R}_{+}^{2}\right)$ such that $B(U, \psi)=\ell(\psi)$ for all $\psi \in \dot{H}^{1}\left(\mathbf{R}_{+}^{2}\right)$, where

$$
\ell(\psi)=-\int_{\mathbf{R}} \phi(x) \psi(x) d x .
$$

Details of this construction for the unbounded domain $\mathbf{R}_{+}^{2}$ can be found in 4, Lemma 1.2]. This function $U$ solves equation (11) and has conormal derivative $\phi$ at the boundary, in a weak sense.

We say that the BVP (Neu- $A, p)$ is well posed in the $\dot{H}^{1}$ sense if for all sufficiently smooth and localised $\phi$ with $\int \phi=0$, the solution $U$ constructed above has quantitative bound

$$
\left\|\widetilde{N}_{*}(\nabla U)\right\|_{L_{p}(\mathbf{R})} \leq C_{p}\|\phi\|_{L_{p}(\mathbf{R})} .
$$

$L_{\infty}\left(L_{p}\right)$ solutions. A different method for constructing a solution $U$ to one of the BVP's above is the boundary equation method. We are given a kernel function $K(t, x ; y)$ which for each $y \in \mathbf{R}$ satisfies equation (1) in the variables $(t, x) \in \mathbf{R}_{+}^{2}$. From this we obtain, for each auxiliary function $h(y)$ on the boundary, a function

$$
U(t, x):=\int_{\mathbf{R}} K(t, x ; y) h(y) d y
$$

solving equation (1) in $\mathbf{R}_{+}^{2}$. Taking the appropriate trace of $U$, depending on which boundary condition $U$ is supposed to satisfy, we get an equation $g=T(h)$, where $g$ denotes either $u, u^{\prime}$ or $\phi$. If the operator $T: L_{p}(\mathbf{R}) \rightarrow L_{p}(\mathbf{R})$ is an isomorphism, then we can solve the equation for $h$ and from this construct a solution $U$.

Obviously there is some freedom of choice for the kernel function $K(t, x ; y)$. In this paper, we shall use the boundary equation method from Auscher, Axelsson and Hofmann 2]. The Cauchy integral method used here for the Neumann and regularity problems actually uses a vector valued kernel $K(t, x ; y)$, and constructs the gradient vector field $F=\nabla U$ rather than $U$ itself. With some abuse of notation (as the invertibility of the boundary equation may depend on the choice of kernel $K)$, we shall say that the BVP's are well posed in the $L_{\infty}\left(L_{p}\right)$ sense, referring to the norm $\sup _{t>0}\|U(t, \cdot)\|_{p}$ for solutions, if this method gives rise to an $L_{p}$ invertible boundary equation.

In this paper we shall prove the following surprising, in view of Theorem 1.1 result.

Theorem 1.2. The boundary equation method of [2] yields the following result for coefficients $A_{k}$. 
The Dirichlet problem $\left(\right.$ Dir- $\left.A_{k}, p\right)$ is well posed in the $L_{\infty}\left(L_{p}\right)$ sense if $k \neq$ $\tan \left(\frac{\pi}{2 q}\right)$, where $1 / q=1-1 / p$. In this case, the solution $U_{t}(x)=U(t, x)$ has bound $\left\|N_{*}(U)\right\|_{p} \leq C\|u\|_{p}$ and convergence $\left\|U_{t}-u\right\|_{p} \rightarrow 0$ as $t \rightarrow 0^{+}$.

The regularity problem $\left(\right.$ Reg- $\left.A_{k}, p\right)$ is well posed in the $L_{\infty}\left(L_{p}\right)$ sense if $k \neq$ $-\tan \left(\frac{\pi}{2 p}\right)$. In this case, the solution $F_{t}(x)=F(t, x)$ has bound $\left\|N_{*}(F)\right\|_{p} \leq C\left\|u^{\prime}\right\|_{p}$ and convergence $\left\|F_{t}-f\right\|_{p} \rightarrow 0$ as $t \rightarrow 0^{+}$, where $f_{1}=u^{\prime}$.

The Neumann problem $\left(\mathrm{Neu}-A_{k}, p\right)$ is well posed in the $L_{\infty}\left(L_{p}\right)$ sense if $k \neq$ $\tan \left(\frac{\pi}{2 p}\right)$. In this case, the solution $F_{t}(x)=F(t, x)$ has bound $\left\|N_{*}(F)\right\|_{p} \leq C\|\phi\|_{p}$ and convergence $\left\|F_{t}-f\right\|_{p} \rightarrow 0$ as $t \rightarrow 0^{+}$, where $f_{0}+k \operatorname{sgn}(x) f_{1}=\phi$.

The reason for these seemingly contradictory results is that the two methods construct different solutions, for some $k$. To illustrate this phenomenon, we study in detail the solutions to the Dirichlet problem in section 4 . We derive the following explicit expression for the solution to the Dirichlet problem with the boundary equation method:

$$
U(t, x)=\frac{1}{\pi} \int_{\mathbf{R}} \frac{2 x t y+|y|^{-\alpha} \operatorname{Im}\left\{(|x|+i t)^{\alpha+1}\left(y^{2}-(|x|-i t)^{2}\right)\right\}}{\left(t^{2}+(x-y)^{2}\right)\left(t^{2}+(x+y)^{2}\right)} u(y) d y,
$$

where $(t, x) \in \mathbf{R}_{+}^{2}$. Here $\tan (\pi \alpha / 2)=k$, and $\alpha \in(1 / q-2,1 / q)$ is the branch obtained with the boundary equation method. Denote the Poisson kernel in (3) by $P_{\alpha}(t, x ; y)$. In Figure 1 the harmonic measures $P_{\alpha}(0.5,1 ; \cdot)$ are plotted for some values of $\alpha$.

On the other hand, for sufficiently smooth and localised boundary data, the $\dot{H}^{1}$ solution to the Dirichlet problem is given by (3), but with the branch $\alpha \in(-1,1)$. For $k>\tan \left(\frac{\pi}{2 q}\right)$, the $\dot{H}^{1}$ solution thus differs from the $L_{\infty}\left(L_{p}\right)$ solution, and we note the following.

- The $\dot{H}^{1}$ solution uses the branch $\alpha \in(1 / q, 1)$. Here the kernel $P_{\alpha}(t, x ; y)$ is always positive, but it does not satisfy the reverse Hölder estimates $B_{q}$ equivalent to the estimate (2), since $P_{\alpha}(t, x ; y) \sim|y|^{-\alpha}$ around $y=0$. The solution does not belong to $L_{p}\left(\mathbf{R}_{x}\right)$ for any fixed $t>0$, because of the slow decay $P_{\alpha}(t, x ; y) \sim|x|^{\alpha-1}$ when $x \rightarrow \infty$.

- The $L_{\infty}\left(L_{p}\right)$ solution uses the branch $\alpha \in(-2+1 / q,-1)$. Here the kernel $P_{\alpha}(t, x ; y)$ is not always positive but does satisfy the reverse Hölder estimates $B_{q}$, since also $P_{\alpha}(t, x ; y) \sim|y|^{-\alpha-2}$ when $y \rightarrow \infty$. The solution is not $\dot{H}^{1}$ up to the boundary in a neighborhood of the origin, not even for smooth boundary data.

The reason why a signed harmonic measure is possible for $\alpha \in(-2+1 / q,-1)$, without contradicting the maximum principle, is that the solution, even for $0 \leq$ $u \in C_{0}^{\infty}(\mathbf{R})$, satisfies $\lim _{t \rightarrow 0^{+}} U(t, 0)=-\infty$. This prevents the $L_{\infty}$ approximation needed for applying the maximum principle. Indeed, (3) shows that

$$
U(t, 0)=\frac{\cos (\pi \alpha / 2)}{\pi} \int_{\mathbf{R}} \frac{t^{1+\alpha}|y|^{-\alpha}}{t^{2}+y^{2}} u(y) d y .
$$

We summarise the main point of this paper. When considering BVP's with nonsymmetric coefficients, it is important to specify which solution is meant. When the $\dot{H}^{1}$ solution to the Dirichlet problem does not satisfy (2), there can still exist another solution $U_{t}(x)=U(t, x)$, which has bound $\left\|N_{*}(U)\right\|_{p}<\infty$ and $L_{p}$ trace $\left\|U_{t}-u\right\|_{p} \rightarrow 0$ as $t \rightarrow 0$. 

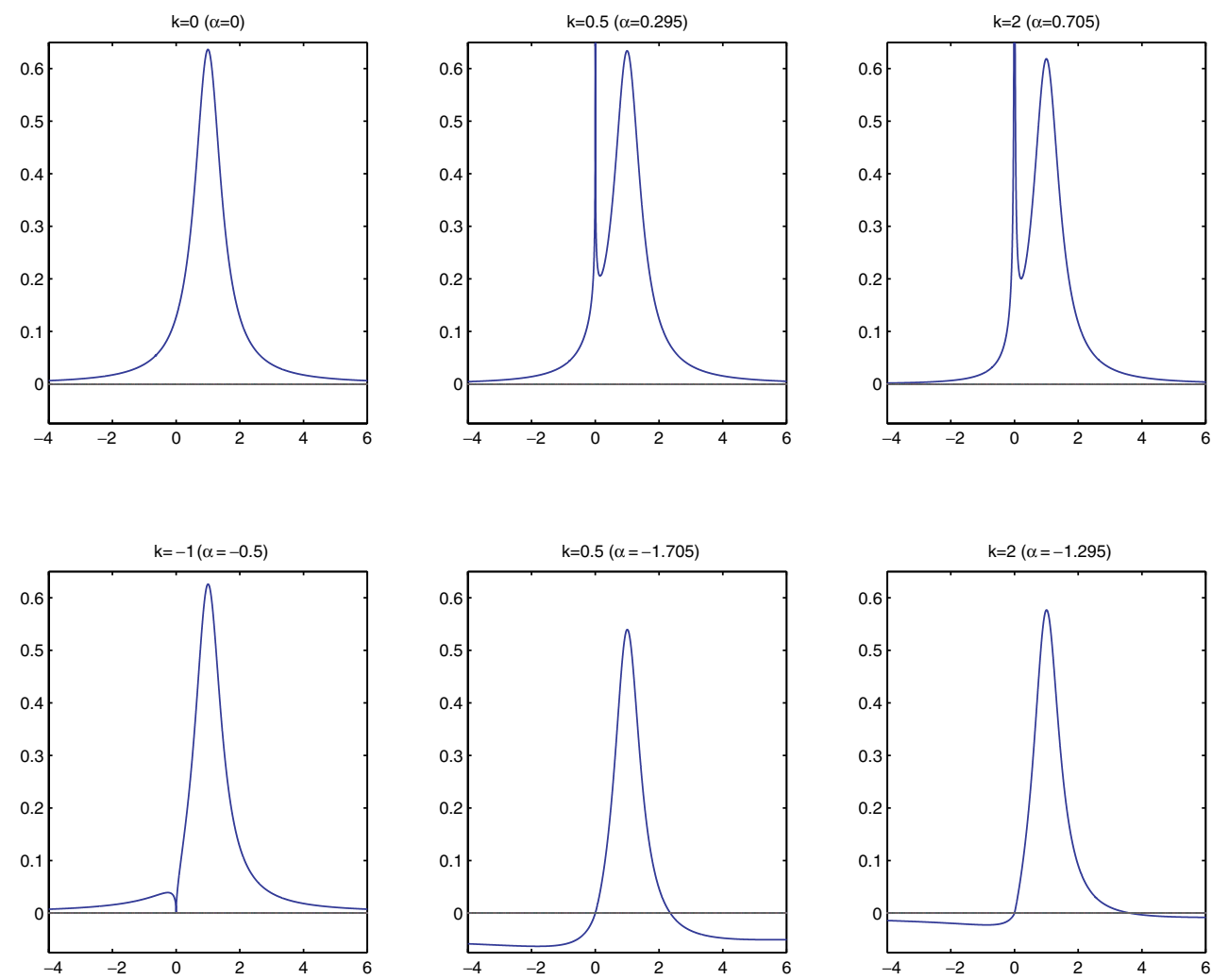

Figure 1

\section{Computation of Cauchy integrals}

In this section we explicitly calculate the basic operators we need in order to solve the BVP's with the boundary equation method from 2]. As in 2, equation (1.5)], we rewrite equation (11) for $U$ as the equivalent first order system $\operatorname{div} A_{k} F=0$ and $\operatorname{curl} F=0$ for the vector field $F=\nabla U$. Solving for the vertical derivative, this first order system reads

$$
\partial_{t} F+\left[\begin{array}{cc}
k\left(\operatorname{sgn}(x) \partial_{x}-\partial_{x} \operatorname{sgn}(x)\right) & \partial_{x} \\
-\partial_{x} & 0
\end{array}\right] F=0 .
$$

Throughout this paper we shall write $f_{0} e_{0}+f_{1} e_{1}$ as $\left[\begin{array}{ll}f_{0} & f_{1}\end{array}\right]^{t}$. The tangential matrix operator in (5) will be denoted by $T_{k}$, and it is seen to be a self-adjoint operator in $L_{2}(\mathbf{R})$, with domain

$$
\mathrm{D}\left(T_{k}\right):=\left\{f_{0} e_{0}+f_{1} e_{1} ; f_{0} \in H^{1}(\mathbf{R}), f_{1}-k \operatorname{sgn}(x) f_{0} \in H^{1}(\mathbf{R})\right\} .
$$

To solve the BVP's, we need to calculate certain operators in the functional calculus of the self-adjoint operator $T_{k}$; in particular we need the following result.

Theorem 2.1. The Cauchy (singular) integral operators for $A_{k}$ are

$$
\operatorname{sgn}\left(T_{k}\right) f(x)=\frac{1}{\pi}\left[\begin{array}{c}
- \text { p.v. } \int \frac{f_{1}(y)}{x-y} d y \\
\text { p.v. } \int \frac{f_{0}(y)}{x-y} d y
\end{array}\right]-\frac{1}{\pi} \frac{k}{1+k^{2}}\left[\begin{array}{c}
\int \frac{f_{0}(y)+k \operatorname{sgn}(y) f_{1}(y)}{|x+| y \mid} d y \\
\operatorname{sgn}(x) \int \frac{k f_{0}(y)-\operatorname{sgn}(y) f_{1}(y)}{|x|+|y|} d y
\end{array}\right]
$$


and

$$
\begin{aligned}
& e^{-t\left|T_{k}\right|} \chi_{+}\left(T_{k}\right) f(x)=\frac{1}{2 \pi}\left[\begin{array}{l}
\int \frac{t f_{0}(y)-(x-y) f_{1}(y)}{t^{2}+(x-y)^{2}} d y \\
\int \frac{t f_{1}(y)+(x-y) f_{0}(y)}{t^{2}+(x-y)^{2}} d y
\end{array}\right] \\
&+\frac{1}{2 \pi} \frac{k}{1+k^{2}}\left[\begin{array}{c}
-\int \frac{t\left(k f_{0}(y)-\operatorname{sgn}(y) f_{1}(y)\right)+(|x|+|y|)\left(f_{0}(y)+k \operatorname{sgn}(y) f_{1}(y)\right)}{t^{2}+(|x|+|y|)^{2}} d y \\
\operatorname{sgn}(x) \int \frac{t\left(f_{0}(y)+k \operatorname{sgn}(y) f_{1}(y)\right)+(|x|+|y|)\left(-k f_{0}(y)+\operatorname{sgn}(y) f_{1}(y)\right)}{t^{2}+(|x|+|y|)^{2}} d y
\end{array}\right]
\end{aligned}
$$

Here $\chi_{ \pm}(z)$ denotes the characteristic function of the right/left complex half plane. We write $\operatorname{sgn}(z):=\chi_{+}(z)-\chi_{-}(z)$ and $|z|:=z \operatorname{sgn}(z)$, for $z \in \mathbf{C}$. Note that $|z|$ does not denote absolute value for non-real $z$.

Lemma 2.2. For non-real $i \lambda \in \mathbf{C}$, the resolvent $\left(i \lambda-T_{k}\right)^{-1} f=u$ is given by

$$
\begin{aligned}
& {\left[\begin{array}{l}
u_{0}(x) \\
u_{1}(x)
\end{array}\right]=\frac{\operatorname{sgn} \lambda}{2}\left[\begin{array}{l}
\int e^{-|\lambda(x-y)|}\left(-i f_{0}(y)+\operatorname{sgn}(\lambda(x-y)) f_{1}(y)\right) d y \\
\int e^{-|\lambda(x-y)|}\left(-\operatorname{sgn}(\lambda(x-y)) f_{0}(y)-i f_{1}(y)\right) d y
\end{array}\right]} \\
& +e^{-|\lambda x|} \frac{k}{2(1-i k \operatorname{sgn}(\lambda))}\left[\begin{array}{c}
i \int e^{-|\lambda y|}\left(-i f_{0}(y)-\operatorname{sgn}(\lambda y) f_{1}(y)\right) d y \\
\operatorname{sgn}(\lambda x) \int e^{-|\lambda y|}\left(-i f_{0}(y)-\operatorname{sgn}(\lambda y) f_{1}(y)\right) d y
\end{array}\right] .
\end{aligned}
$$

To prove the lemma, we need to solve $\left(i \lambda-T_{k}\right) u=f$ for $u$. Thus we are looking for $u$ such that

$$
\left\{\begin{array}{l}
u_{0}^{\prime}=-i \lambda u_{1}+f_{1} \\
u_{1}^{\prime}=i \lambda u_{0}-f_{0}
\end{array}\right.
$$

for $x \neq 0$, and such that $u \in \mathrm{D}\left(T_{k}\right)$, i.e. $u_{0}$ is continuous at $x=0$ whereas

$$
u_{1}(0+)-u_{1}(0-)=2 k u_{0}(0) .
$$

Multiplying the system of equations by $M=[-i, 1 ; 1,-i]$ gives the diagonal system

$$
\left\{\begin{array}{l}
v_{0}^{\prime}=-\lambda v_{0}+g_{0}, \\
v_{1}^{\prime}=\lambda v_{1}+g_{1},
\end{array}\right.
$$

for $v=M u$ and $g:=[-1,-i ; i, 1] f$. Integrating these equations and using the jump condition at $x=0$ gives the formula in the lemma.

Lemma 2.3. The operators $P_{t}=\left(1+t^{2} T_{k}^{2}\right)^{-1}$ and $Q_{t}=t T_{k}\left(1+t^{2} T_{k}^{2}\right)^{-1}$, for $t>0$, are

$$
\begin{aligned}
P_{t} f(x)= & {\left[\begin{array}{c}
\int \frac{1}{2 t}\left(e^{-|x-y| / t} f_{0}(y)+\frac{k}{1+k^{2}} e^{-(|x|+|y|) / t}\left(-k f_{0}(y)+\operatorname{sgn}(y) f_{1}(y)\right)\right) d y \\
\int \frac{1}{2 t}\left(e^{-|x-y| / t} f_{1}(y)+\frac{k \operatorname{sgn}(x)}{1+k^{2}} e^{-(|x|+|y|) / t}\left(f_{0}(y)+k \operatorname{sgn}(y) f_{1}(y)\right)\right) d y
\end{array}\right], } \\
Q_{t} f(x)= & {\left[\begin{array}{c}
\int-\frac{1}{2 t} \operatorname{sgn}(x-y) e^{-|x-y| / t} f_{1}(y) d y \\
\int \frac{1}{2 t} \operatorname{sgn}(x-y) e^{-|x-y| / t} f_{0}(y) d y
\end{array}\right] } \\
- & {\left[\begin{array}{c}
\int \frac{1}{2 t} \frac{k}{1+k^{2}} e^{-(|x|+|y|) / t}\left(f_{0}(y)+k \operatorname{sgn}(y) f_{1}(y)\right) d y \\
\int \frac{1}{2 t} \frac{k \operatorname{sgn}(x)}{1+k^{2}} e^{-(|x|+|y|) / t}\left(k f_{0}(y)-\operatorname{sgn}(y) f_{1}(y)\right) d y
\end{array}\right] . }
\end{aligned}
$$

This follows from Lemma 2.2 and the formulae

$$
\begin{aligned}
P_{t} & =\frac{1}{2 i t}\left(\left(\frac{1}{i t}-T_{k}\right)^{-1}-\left(\frac{1}{-i t}-T_{k}\right)^{-1}\right) \text { and } \\
Q_{t} & =-\frac{1}{2 t}\left(\left(\frac{1}{i t}-T_{k}\right)^{-1}+\left(\frac{1}{-i t}-T_{k}\right)^{-1}\right) .
\end{aligned}
$$


We are now in a position to prove Theorem 2.1. As in [2, Section 2.3], we use the Dunford functional calculus formula

$$
b\left(T_{k}\right)=\frac{1}{2 \pi i} \int_{\gamma} b(\lambda)\left(\lambda-T_{k}\right)^{-1} d \lambda,
$$

where $\gamma$ is the boundary of a double sector around $\mathbf{R} \backslash\{0\}$. Using $b(z)=\operatorname{sgn}(z)$ and $b(z)=e^{-t|z|} \chi_{+}(z)$ respectively, together with Lemma 2.2. gives the formulae in Theorem 2.1. However, the computations can be somewhat simplified by choosing a degenerate contour of integration along the imaginary axis. In this case the Dunford formulae become

$$
\begin{aligned}
\operatorname{sgn}\left(T_{k}\right) & =\frac{2}{\pi} \int_{0}^{\infty} Q_{s} \frac{d s}{s}, \\
e^{-t\left|T_{k}\right|} \chi_{+}\left(T_{k}\right) & =\frac{1}{\pi} \int_{0}^{\infty}\left(Q_{s} \cos (t / s)+P_{s} \sin (t / s)\right) \frac{d s}{s} .
\end{aligned}
$$

Changing the order of integration for $s$ and $y$ here, and using the fact that

$$
\int_{0}^{\infty} \frac{1}{s} e^{-x / s} e^{i t / s} \frac{d s}{s}=\frac{x+i t}{x^{2}+t^{2}}
$$

gives the desired formulae. Some of the above computations are of course formal. However, they can be justified for example with arguments like those in [5].

\section{Solvability of Boundary EQUATiOns}

In this section we use the Cauchy integrals from Theorem 2.1 to solve BVP's, following the boundary equation method described in [2].

Definition 3.1. Let $E_{k}^{ \pm} h:=\chi_{ \pm}\left(T_{k}\right) h$ be the Hardy projections, with the associated Cauchy singular integral operator $E_{k} h:=\operatorname{sgn}\left(T_{k}\right) h$. Let the Cauchy extension operators be $\left(C_{k}^{ \pm} h\right)(t, x):=\left(e^{\mp t\left|T_{k}\right|} E_{k}^{ \pm} h\right)(x), \pm t>0$.

Note that $E_{k}=E_{k}^{+}-E_{k}^{-}$and conversely $E_{k}^{ \pm}=\frac{1}{2}\left(I \pm E_{k}\right)$. Given a function $h: \mathbf{R} \rightarrow \mathbf{C}^{2}$ on the boundary, applying the Cauchy extension $C_{k}^{+}$gives a vector field $F(t, x)=C_{k}^{+} h(x)$ in $\mathbf{R}_{+}^{2}$. This is our ansatz for the regularity and Neumann problems. Indeed, the vertical derivative of $F=e^{-t\left|T_{k}\right|} E_{k}^{+} h$ is

$$
\partial_{t} F=-\left|T_{k}\right| e^{-t\left|T_{k}\right|} \chi_{+}\left(T_{k}\right) h=-T_{k}\left(e^{-t\left|T_{k}\right|} \chi_{+}\left(T_{k}\right) h\right)=-T_{k} F .
$$

Thus $F$ satisfies (5) or, equivalently, the first order system $\operatorname{div} A_{k} F=0$ and $\operatorname{curl} F=$ 0 . This means that $F$ is a gradient vector field $F=\nabla U$, with potential $U$ that solves (11).

On the other hand, to solve the Dirichlet problem we make use of the fact that, due to the $t$-independence of the coefficients $A_{k}$,

$$
0=\partial_{t}\left(\operatorname{div} A_{k}(x) F\right)=\operatorname{div} A_{k}(x)\left(\partial_{t} F\right)=\operatorname{div} A_{k}(x) \nabla F_{0},
$$

i.e. the normal component $F_{0}=e_{0} \cdot C_{k}^{+} h$ of the Cauchy extension satisfies equation (11). This will be our ansatz for the Dirichlet problem.

Lemma 3.2. Let $h \in L_{p}\left(\mathbf{R} ; \mathbf{C}^{2}\right)$. Then we have bounds $\left\|E_{k} h\right\|_{p} \leq C\|h\|_{p}$ and $\left\|N_{*}\left(C_{k}^{+} h\right)\right\|_{p} \leq C\|h\|_{p}$, and convergence

$$
\left\|C_{k}^{+} h(t, \cdot)-E_{k}^{+} h\right\|_{p} \longrightarrow 0, \quad t \longrightarrow 0^{+} .
$$


Proof. By breaking up the integrals into $\int_{\mathbf{R}}=\int_{\mathbf{R}_{+}}+\int_{\mathbf{R}_{-}}$for the second terms in Theorem 2.1, we see that we can write each component of $C_{k}^{+} h$ as

$$
\chi_{+}(x)\left(\frac{t}{t^{2}+x^{2}} * \tilde{h}_{1}(x)+\frac{x}{t^{2}+x^{2}} * \tilde{h}_{2}(x)\right)+\chi_{-}(x)\left(\frac{t}{t^{2}+x^{2}} * \tilde{h}_{3}(x)+\frac{x}{t^{2}+x^{2}} * \tilde{h}_{4}(x)\right),
$$

for some $L_{p}$ functions $\tilde{h}_{i}$. Similarly $E_{k} h$ can be expressed in terms of the Hilbert transform. The lemma now follows from well known $L_{p}$ bounds and convergence for these convolution operators.

Definition 3.3. Let the double layer potential type operator for $A_{k}$ be

$$
K f(x):=\operatorname{sgn}(x) \frac{1}{\pi} \text { p.v. } \int \frac{f(y)}{x-y} d y-\frac{1}{\pi} \int \frac{f(y)}{|x|+|y|} d y,
$$

acting boundedly in $L_{p}(\mathbf{R} ; \mathbf{C})$.

Proposition 3.4. The boundary equation method from [2] constructs solutions to the BVP's as follows.

- A solution to the Neumann problem $\left(\mathrm{Neu}-A_{k}, p\right)$ is given by

$$
\nabla U(t, x)=C_{k}^{+}\left[\begin{array}{ll}
\psi & 0
\end{array}\right]^{t},
$$

where $\phi=\frac{1}{2}(I+k K) \psi$.

- A solution to the regularity problem $\left(\operatorname{Reg}-A_{k}, p\right)$ is given by

$$
\nabla U(t, x)=C_{k}^{+}[-k \operatorname{sgn}(x) \psi(x) \quad \psi(x)]^{t},
$$

where $\operatorname{sgn}(x) u^{\prime}(x)=\frac{1}{2}(I-k K)(\operatorname{sgn}(y) \psi(y))$.

- A solution to the Dirichlet problem $\left(\right.$ Dir- $\left.A_{k}, p\right)$ is given by

$$
U(t, x)=e_{0} \cdot C_{k}^{+}\left[\begin{array}{ll}
\psi(x) & k \operatorname{sgn}(x) \psi(x)
\end{array}\right]^{t},
$$

where $u=\frac{1}{2}(I+k K)^{*} \psi$.

Proof. Lemma 3.2 shows that the Cauchy extension has trace $\lim _{t \rightarrow 0^{+}} C_{k}^{+} h=$ $\frac{1}{2}\left(h+E_{k} h\right)$. To solve the Neumann problem, we write $h=\left[\begin{array}{ll}\psi & 0\end{array}\right]^{t}$ for the given ansatz. Using the first formula in Theorem 2.1 for $E_{k}$, the Neumann boundary condition becomes

$$
\begin{aligned}
& \phi=e_{0} \cdot\left(A_{k} \frac{1}{2}\left(h+E_{k} h\right)\right) \\
& =\frac{1}{2}\left[\begin{array}{ll}
1 & k \operatorname{sgn}(x)
\end{array}\right]\left(\left[\begin{array}{l}
\psi \\
0
\end{array}\right]+\frac{1}{\pi}\left[\begin{array}{c}
0 \\
\text { p.v. } \int \frac{\psi(y)}{x-y} d y
\end{array}\right]-\frac{1}{\pi} \frac{k}{1+k^{2}}\left[\begin{array}{c}
\int \frac{\psi(y)}{|x|+|y|} d y \\
\operatorname{sgn}(x) \int \frac{k \psi(y)}{|x|+|y|} d y
\end{array}\right]\right) \\
& =\frac{1}{2}(\psi+k K \psi),
\end{aligned}
$$

as stated. Similar calculations for the Dirichlet boundary conditions give the stated boundary equations.

Remark 3.5. For motivation of the choices made for the ansatzes, we refer to 2 . In the language used there, we express $\hat{N}_{A}^{-} E_{A}^{+} \hat{N}_{A}^{-}, \hat{N}_{A}^{+} E_{A}^{+} \hat{N}_{A}^{+}$and $\check{N}_{A}^{-} E_{A}^{+} \check{N}_{A}^{-}$in terms of the operator $K$. The idea is to compress the Cauchy integral to a suitable subspace in $L_{p}(\mathbf{R})$ which is complementary to the null space of the boundary condition under consideration. We use the projections $\hat{N}_{A}^{-}=[1, k \operatorname{sgn}(x) ; 0,0]$, $\hat{N}_{A}^{+}=[0,-k \operatorname{sgn}(x) ; 0,1]$ and $\check{N}_{A}^{-}=[1,0 ; k \operatorname{sgn}(x), 0]$, which project onto the complementary subspace along the null space. 
To prove Theorem 1.2, we see that it suffices to investigate the $L_{p}$ spectrum of $K$. We first note that $K=K_{0} \oplus K_{0}$ in the splitting $L_{p}(\mathbf{R})=L_{p}\left(\mathbf{R}_{+}\right) \oplus L_{p}\left(\mathbf{R}_{-}\right)$, where $K_{0}$ is the operator in the following proposition.

Proposition 3.6. The operator

$$
K_{\alpha} f(x):=\frac{2}{\pi} \text { p.v. } \int_{0}^{\infty} \frac{x^{\alpha} y^{1-\alpha}}{x^{2}-y^{2}} f(y) d y
$$

is bounded on $L_{p}\left(\mathbf{R}_{+} ; \mathbf{C}\right)$ if and only if $\alpha \in(-1 / p, 2-1 / p)$. Furthermore, if $\alpha \in(-1 / p, 2-1 / p)$ and $k=\tan \left(\frac{\pi}{2} \alpha\right)$, then $I-k K_{0}$ is invertible in $L_{p}$ with inverse

$$
\left(I-k K_{0}\right)^{-1}=\frac{1}{1+k^{2}}\left(I+k K_{\alpha}\right) .
$$

Proof. We use the isometry

$$
U: L_{p}\left(\mathbf{R}_{+}\right) \longrightarrow L_{p}(\mathbf{R}), \quad f(x) \longmapsto e^{t / p} f\left(e^{t}\right) .
$$

A calculation shows that $U K_{\alpha} U^{-1}=\widetilde{K}_{\alpha+1 / p}$ for the convolution operator

$$
\widetilde{K}_{\gamma} g:=\frac{2}{\pi} \text { p.v. } \frac{e^{\gamma t}}{e^{2 t}-1} * g .
$$

By standard singular integral theory $\widetilde{K}_{\gamma}$ is bounded, on all $L_{p}$ spaces, if and only if $\gamma \in(0,2)$. This proves the boundedness result for $K_{\alpha}$.

To verify the inverse relation, we need to show that

$$
\left(I-k \widetilde{K}_{1 / p}\right)\left(I+k \widetilde{K}_{\alpha+1 / p}\right)=1+k^{2} .
$$

Applying the Fourier transform, this amounts to showing that

$$
\left(1-k i \frac{1+z}{1-z}\right)\left(1+k i \frac{1+z e^{i \pi \alpha}}{1-z e^{i \pi \alpha}}\right)=1+k^{2},
$$

where $z:=e^{\pi(\xi+i / p)}$. This is verified by using the relation $e^{i \pi \alpha}=(1+i k) /(1-$ $i k)$.

Proof of Theorem 1.2. Proposition 3.6 shows in particular that $I-k K_{0}$ is invertible in $L_{p}(\mathbf{R})$ if $k \neq-\tan \left(\frac{\pi}{2 p}\right)$. Therefore the boundary equations derived in Proposition 3.4 are invertible under the hypotheses in Theorem 1.2 . The bounds and convergence of the solutions follow from Lemma 3.2 .

\section{The HaRmonic Measure}

The kernels of the solution operators in Proposition 3.4 can now be calculated. Here we only calculate the harmonic measure $P_{\alpha}(t, x ; \cdot) d y$, where $P_{\alpha}$ denotes the Poisson kernel, i.e. the kernel of the solution operator for the Dirichlet problem. The regularity and Neumann problems can be further studied in much the same way. According to Proposition 3.4 and Theorem 2.1, a solution to the Dirichlet problem is

$$
U(t, x)=\frac{1}{2 \pi} \int\left(\frac{t-(x-y) k \operatorname{sgn}(y)}{t^{2}+(x-y)^{2}}-k \frac{|x|+|y|}{t^{2}+(|x|+|y|)^{2}}\right) \psi(y) d y,
$$

with

$$
\psi( \pm y)=\frac{2}{1+k^{2}}\left(u( \pm y)-k \frac{2}{\pi} \text { p.v. } \int_{0}^{\infty} \frac{y^{1+\alpha} z^{-\alpha}}{y^{2}-z^{2}} u( \pm z) d z\right), \quad y>0,
$$

where $k=\tan \left(\frac{\pi}{2} \alpha\right)$ and $\alpha \in(1 / q-2,1 / q)$, according to Proposition 3.6. 
Composing these two operators yields the formula (3) for the harmonic measure. The calculation makes use of the following residue calculus formula.

Lemma 4.1. For $\alpha \in(-2,1)$ and $t, z>0$, write

$$
I_{\alpha, \beta, \gamma}(t, x, z):=\text { p.v. } \int_{0}^{\infty} \frac{\gamma t+\beta(y-x)}{t^{2}+(y-x)^{2}} \frac{y^{1+\alpha}}{y^{2}-z^{2}} d y .
$$

Then, with $\arg (x+i t) \in(0, \pi)$, we have

$$
\begin{gathered}
2 \frac{k}{\pi} z^{-\alpha} I_{\alpha, \beta, \gamma}(t, x, z)=\frac{k^{2}-1}{2} \frac{\gamma t-\beta(x-z)}{t^{2}+(x-z)^{2}}-\frac{k^{2}+1}{2} \frac{\gamma t-\beta(x+z)}{t^{2}+(x+z)^{2}} \\
-\operatorname{Re}\left(\frac{(1-i k)^{2}(\beta-i \gamma)(x+i t)^{1+\alpha}}{(x+i t)^{2}-z^{2}}\right) z^{-\alpha} .
\end{gathered}
$$

From section 3 it is clear that $u \mapsto \psi \mapsto U$ gives a solution to (Dir- $\left.A_{k}, p\right)$ when $\alpha \in(1 / q-2,1 / q)$, and that $U_{t} \rightarrow u$ in $L_{p}(\mathbf{R})$ when $t \rightarrow 0^{+}$. We now investigate the Poisson integral formula (3) for more general $\alpha$. First note that $P_{\alpha}(t, x ; \cdot)$ is not locally $L_{1}$ if $\alpha \geq 1$, and if $\alpha \leq-2$, then it does not decay at $\infty$. For these reasons, we only consider $\alpha \in(-2,1)$. We shall now consider the branch $\alpha \in(-1,1)$ in (3) and show that this gives the $\dot{H}^{1}$ solution to $\left(\operatorname{Dir}-A_{k}, p\right)$. For the rest of the section, we assume that $u \in C_{0}^{\infty}(\mathbf{R})$ and $\alpha \in(-1,1)$.

From (6), it is seen that $\psi \in \dot{H}^{1 / 2}(\mathbf{R})$ if $u \in C_{0}^{\infty}(\mathbf{R})$. Moreover, we shall use the fact that $\tilde{\psi}:=\psi-\psi(0) e^{-|x|}$ satisfies $\tilde{\psi} \in \dot{H}^{1 / 2}(\mathbf{R})$ and has bound $|\tilde{\psi}(x)| \leq$ $C \min (|x|, 1 /|x|)^{\gamma}$ for some $\gamma>0$.

Lemma 4.2. If $u \in C_{0}^{\infty}(\mathbf{R})$ and $\alpha \in(-1,1)$, then

$$
\iint_{\mathbf{R}_{+}^{2}}|\nabla U(t, x)|^{2} d t d x<\infty
$$

Proof. Write $h:=\left[\begin{array}{ll}\psi(x) & k \operatorname{sgn}(x) \psi(x)\end{array}\right]^{t}$ for the ansatz in Proposition 3.4, and note that

$$
\nabla U=\nabla\left(e_{0} \cdot C_{k}^{+} h\right)=\partial_{t} C_{k}^{+} h=-T_{k} C_{k}^{+} h=-E_{k}^{+}\left|T_{k}\right| e^{-t\left|T_{k}\right|} h .
$$

Since $T_{k}$ is self-adjoint, it satisfies $L_{2}$ quadratic estimates. Therefore

$$
\begin{aligned}
\iint_{\mathbf{R}_{+}^{2}} \mid \nabla U(t, & x)\left.\right|^{2} d t d x=\int_{0}^{\infty}\left\|E_{k}^{+}\left|T_{k}\right| e^{-t\left|T_{k}\right|} h\right\|_{2}^{2} d t \\
& \leq \int_{0}^{\infty}\left\|\left|t T_{k}\right|^{1 / 2} e^{-t\left|T_{k}\right|}\left(\left|T_{k}\right|^{1 / 2} h\right)\right\|_{2}^{2} \frac{d t}{t} \approx\left\|\left|T_{k}\right|^{1 / 2} h\right\|_{2}^{2} \\
& \approx \int_{0}^{\infty}\left\|\left|t T_{k}\right|^{3 / 2}\left(1+t^{2} T_{k}^{2}\right)^{-1}\left(\left|T_{k}\right|^{1 / 2} h\right)\right\|_{2}^{2} \frac{d t}{t}=\int_{0}^{\infty}\left\|h-P_{t} h\right\|_{2}^{2} \frac{d t}{t^{2}}
\end{aligned}
$$

since $|z|^{1 / 2} e^{-|z|}$ and $|z|^{3 / 2}\left(1+z^{2}\right)^{-1}$ decay at 0 and $\infty$. Lemma 2.3 shows that

$$
\left\|h-P_{t} h\right\|_{2}^{2}=\left\|\psi-p_{t} * \psi\right\|_{2}^{2}+k^{2}\left\|\operatorname{sgn}(x) \psi-p_{t} *(\operatorname{sgn}(x) \psi)-2 t\left(\psi, p_{t}\right) \operatorname{sgn}(x) p_{t}\right\|_{2}^{2},
$$

where $p_{t}(x):=\frac{1}{2 t} e^{-|x| / t}$. We now recall that $\psi=\tilde{\psi}+2 \psi(0) p_{1}$, where $\tilde{\psi} \in \dot{H}^{1 / 2}(\mathbf{R})$ and $|\tilde{\psi}(x)| \leq C \min (|x|, 1 /|x|)^{\gamma}$ for some $\gamma>0$, and that

$$
\|f\|_{\dot{H}^{1 / 2}(\mathbf{R})}^{2} \approx \int_{\mathbf{R}} \int_{\mathbf{R}} \frac{|f(x)-f(y)|^{2}}{|x-y|^{2}} d x d y \approx \int_{\mathbf{R}}|\hat{f}(\xi)|^{2}|\xi| d \xi .
$$


Using the first expression for the norm, we verify that $\operatorname{sgn}(x) \tilde{\psi} \in \dot{H}^{1 / 2}(\mathbf{R})$, and by applying Plancherel's theorem and using the second expression, we show that

$$
\int_{0}^{\infty}\left\|f-p_{t} * f\right\|_{2}^{2} \frac{d t}{t^{2}} \leq C\|f\|_{\dot{H}^{1 / 2}(\mathbf{R})}^{2} .
$$

Thus it remains to show that

$$
\int_{0}^{\infty}\left|\left(\tilde{\psi}, p_{t}\right)\right|^{2} \frac{d t}{t}+|\psi(0)|^{2} \int_{0}^{\infty}\left\|\operatorname{sgn}(x) p_{1}-p_{t} *\left(\operatorname{sgn}(x) p_{1}\right)-2 t\left(p_{1}, p_{t}\right) \operatorname{sgn}(x) p_{t}\right\|^{2} \frac{d t}{t^{2}}<\infty .
$$

Here the first term is finite since $\left|\left(\tilde{\psi}, p_{t}\right)\right| \leq C \min (t, 1 / t)^{\gamma}$, and an explicit calculation for the second term gives $\|\cdots\|^{2} \leq C \min \left(t^{2}, 1\right)$, which shows that the second term is finite. This proves the lemma.

Restricting (3) to $x=0$ gives the formula (4). In the quadrants $t>0, \pm x>0$, the equation $\operatorname{div} A_{k} \nabla U=0$ reduces to the Laplace equation. For example, in the first quadrant, the Poisson integral for the Laplace equation yields

$$
\begin{aligned}
U(t, x)=\frac{1}{\pi} \int_{0}^{\infty} \frac{4 x t y}{4 x^{2} t^{2}+\left(x^{2}-t^{2}-y^{2}\right)^{2}} u(y) d y \\
\quad+\frac{1}{\pi} \int_{0}^{\infty} \frac{4 x t s}{4 x^{2} t^{2}+\left(x^{2}-t^{2}+s^{2}\right)^{2}} U(s, 0) d s
\end{aligned}
$$

when $t>0, x>0$. Inserting expression (4) into the second term and calculating the integral

$$
\begin{aligned}
\frac{1}{\pi} & \int_{0}^{\infty} \frac{s^{2+\alpha}}{\left(4 x^{2} t^{2}+\left(x^{2}-t^{2}+s^{2}\right)^{2}\right)\left(s^{2}+y^{2}\right)} d s \\
& =-\frac{1}{2} \frac{1}{\cos (\pi \alpha / 2)} \frac{|y|^{1+\alpha}}{4 x^{2} t^{2}+\left(x^{2}-t^{2}-y^{2}\right)^{2}}+\frac{1}{4 x t} \operatorname{Re}\left((1-i k) \frac{(t+i x)^{1+\alpha}}{(t+i x)^{2}+y^{2}}\right)
\end{aligned}
$$

gives back (3).

It is clear from (7) that $U$ satisfies the Laplace equation in the quadrants $t>0$, $\pm x>0$. Furthermore, calculating $\partial_{x} U(t, 0 \pm)$ from (3) and $\partial_{t} U(t, 0)$ from (4) shows that $\partial_{x} U(t, 0+)-\partial_{x} U(t, 0-)=2 k \partial_{t} U(t, 0)$ for $t>0$. This proves that $\operatorname{div} A_{k} \nabla U=0$ in $\mathbf{R}_{+}^{2}$. Furthermore it is clear from (7) that $U$ has boundary trace $u$ in the weak sense. This proves that (3) gives the $\dot{H}^{1}$ solution to the Dirichlet problem when $\alpha \in(-1,1)$. Finally we note that it follows from (7) and (4) that $P_{\alpha}(t, x ; y) \geq 0$ for all $t>0$ and $x, y \in \mathbf{R}$ when $\alpha \in(-1,1)$.

\section{ACKNOWLEDGMENTS}

The author thanks Pascal Auscher and Steve Hofmann for many interesting discussions on the topic of this paper.

\section{REFERENCES}

[1] Alfonseca, M., Auscher, P., Axelsson, A., Hofmann, S., and Kim, S. Analyticity of layer potentials and $L^{2}$ solvability of boundary value problems for divergence form elliptic equations with complex $L^{\infty}$ coefficients. Preprint.

[2] Auscher, P., Axelsson, A., And Hofmann, S. Functional calculus of Dirac operators and complex perturbations of Neumann and Dirichlet problems. Preprint.

[3] Kenig, C., Koch, H., Pipher, J., And Toro, T. A new approach to absolute continuity of elliptic measure, with applications to non-symmetric equations. Adv. Math. 153, no. 2 (2000), 231-298. MR.1770930(2002f:35071) 
[4] Kenig, C., And Rule, D. The regularity and Neumann problem for non-symmetric elliptic operators. Preprint.

[5] M ${ }^{\mathrm{C}}$ Intosh, A., AND Qian, T. Convolution singular integral operators on Lipschitz curves. In Harmonic analysis (Tianjin, 1988), vol. 1494 of Lecture Notes in Mathematics Springer, Berlin, 1991, pp. 142-162.

Matematiska institutionen, Stockholms universitet, 10691 Stockholm, Sweden

E-mail address: andax@math.su.se 